Concept Note

\title{
Immunity Passport Against COVID 19
}

FEROZA BEGUM ${ }^{1}$ and UPASANA RAY*,2

${ }^{1}$ CSIR-Indian Institute of Chemical Biology, 4, Raja S.C., Mullick Road, Jadavpur, Kolkata 700032 , West Bengal, India

${ }^{2}$ Academy of Scientific and Innovative Research (AcSIR), Ghaziabad 201 002, India

(Received on 03 July 2020; Revised on 06 September 2020; Accepted on 18 September 2020)

\begin{abstract}
We are going through a major pandemic of COVID-19/SARS-CoV-2 currently. Several measures are been tested to either treat the infection or prevent future infection and also further spread of the ongoing infection. Recently, the issue of immunity passports has been in discussion. Immunity passports are supposed to mark an individual as immune to SARSCoV-2. While some supported the idea, many didn't. Here, we have discussed both the pros and cons of the idea of immunity passport.
\end{abstract}

Keywords: Immunity Passport; Covid-19; Immunity; Antibodies

\section{The Idea}

\section{What is an immunity passport and what is the rationale?}

Immunity passport is a certification that would mark an individual immune to SARS-CoV-2 infection (Fraser, 2020). The rationale behind certifying people immune is the production of anti-SARS-CoV-2 antibodies.

\section{Who can be certified?}

It is believed that people who have recovered from COVID 19 and are anti-SARS-CoV-2 antibody positive or the antibody positive asymptomatic individuals can be issued immunity passport.

\section{How immunity passport could be beneficial?}

COVID-19 pandemic has created immense economic crisis and halted activities in various socio-economic sectors of affected countries including India. Prolonged disruption of essential healthcare, transportation, education and other important areas might lead to significant economic loss. India has additionally experienced huge crisis for daily-wage workers, migrant laborers and low-income families.
Thus, strategically devised immunity passport policies or certifications might help return of individuals serologically found to be on safer side to resume duties and thus let the economy recover even though at slower pace.

\section{Problems in the Theory}

All individuals who recover from SARS-CoV-2 should be producing antibodies against this virus. Although this theory may sound theoretically right, there is no evidence that all individuals would produce good quality antibodies or the neutralizing antibodies that would protect them from re-infections. Also, antibodies just like other proteins have half-life and would not persist in the system for long. It's the primed plasma cells (the antibody producing B cells) that would produce and release antibodies when challenged by the virus or the viral proteins against which the B cells learnt to make the antibodies. The problems in issuing immunity passport to these individuals are discussed as follows:

\section{Neutralizing Antibodies}

In a study published in 'The New England journal of Medicine' on September 1, 2020, Gudbjartsson et al.

*Author for Correspondence: E-mail: ray.upasana@gmail.com 
have shown that individuals infected once maintain anti-SARS-CoV-2 antibodies for nearly four months (Gudbjartsson et al., 2020). However, the same authors, as well as other reports, have also discussed that the antibody levels vary among different groups (Gudbjartsson et al., 2020; Zhao J et al., 2020). Antibodies appear to be higher in severe infections than the non-severe infections and asymptomatic individuals. Also, women and smokers had lower antibody levels. It has been shown that a fraction of infected people become antibody negative during the convalescent period (Long et al., 2020). Thus, it is difficult to exactly assure an infected person about the duration for which he/she is protected.

SARS-CoV-2 has many variants/genotypes/ clades (Mercatelli et al., 2020; Brufsky, 2020; Coronaviridae Study Group of the International Committee on Taxonomy of Viruses, 2020; Forster et $a l ., 2020)$. These groups differ from one another at gene levels. As a result, these variants would harbour proteins with similar but not same amino acid sequences at least with respect to few major proteins like the spike, RNA dependent RNA polymerase etc. Such amino acid substitutions or differences would in turn render the immune system incapable of producing antibodies that would neutralize all the variants universally. This might or might not happen. Mere production of antibodies does not imply production of neutralizing antibodies. Neutralization means to be able to stop the virus from gaining entry in its host cells i.e. the virus entry/ infection. B cells might simply be producing antibodies that would bind but would not neutralize the variants other than the one that infected the individual earlier. We see similar problem in case of Dengue.

Since this virus is evolving even within the country re-infection with a different variant will not be surprising. Travelling to a different country or continent would also increase the probability of getting challenged by a different virus type against which the person might not be able to produce neutralizing antibodies.

Also, antibody positive individuals might gain possible false impression to be absolutely safe and might lead to the possibility of these people ignoring public health advice. Lifting restrictions on antibody positive people might thus put them in front of the risk of re-infections after the expiry of their antibody life span which is still an area of research.

\section{Antibody Dependent Enhancement}

There is no evidence that a re-infection would not lead to antibody dependent enhancement (ADE) of the viral infection. Antibody dependent enhancement is a phenomenon by which the antibodies that are not neutralizing antibodies would bind the virus nut instead of neutralizing or inhibiting virus transduction in its host cells would enhance the viral entry by an alternate entry route i.e. via the interaction of antibody's Fc portion and the $\mathrm{Fc}$ receptors present on the cell membrane (Each antibody has two parts: the Fab that binds the antigen and the $\mathrm{Fc}$ that binds the $\mathrm{Fc}$ receptors) (Halstead, 2003). This phenomenon is also reported in case of Dengue and is a big hurdle in the field of vaccine development (Halstead, 2003; Halstead et al., 1977; Rothman et al., 2011). Unless antibodies are cross-neutralizing in nature, they won't have protective properties against a different variant in long run. Thus, elaborate serology is needed.

All these also hold true (Arvin et al., 2020)for asymptomatic individuals positive for antibodies against SARS-CoV-2. The today's asymptomatic might become symptomatic under challenge with a different virus variant later or if such people travel to a country where some other variant is dominating.

\section{Cross-Reactive Antibodies: Blessing or Curse?}

We are exposed to many low-pathogenic coronaviruses (causing mild upper respiratory tract infections) and many of the acute respiratory illnesses are attributed to these viruses (GBD 2015 Disease and Injury Incidence and Prevalence Collaborators, 2015). Grefoni et al. when tested SARS-CoV-2 unexposed donors for possibility of seroreactivity against $\mathrm{HCoV}-\mathrm{OC} 43$ and $\mathrm{HCoV}-\mathrm{NL} 63$ found that all the donors were $\mathrm{IgG}$ seropositive to $\mathrm{HCoV}-\mathrm{OC} 43$ and $\mathrm{HCoV}-\mathrm{NL} 63$ receptor binding domain (RBD). HCoV$\mathrm{OC} 43$ and HCoV-NL63 were used as a representative of betacoronavirus and alphacoronavirus, respectively (Grifoni et al., 2020). However, we do not know if these cross-reactive antibodies from infections from other low-pathogenic coronaviruses would also be cross-neutralizing or cross-protective. To address this issue, more in depth and diverse serological testing is warranted. Moreover, cross-reactive antibodies might 
either be without functional relevance, have crossneutralizing properties or subject an individual to the condition of ADE. If there are cross-neutralizing properties, this would mean cross-protection and thus reduce the severity of SARS-CoV-2 infection. However, if cross-reactive properties links to ADE, then such antibodies might promote more severe infection. Therefore, to determine immunity against SARS-CoV-2 surveillance with respect to crossreactive antibodies need to be performed and their functional roles need to be determined.

\section{Conclusion}

What will happen to rest of the people who are negative for anti-SARS-CoV-2 antibodies? There is no evidence that herd immunity will develop. Even if

\section{References}

Fraser B (2020) Chile plans controversial COVID-19 certificates Lancet 3951473

Gudbjartsson D F, Norddahl G L, Melsted P et al. (2020) Humoral Immune Response to SARS-CoV-2 in Iceland [published online ahead of print]

Zhao J, Yuan Q, Wang H, et al. (2019) Antibody responses to SARS-CoV-2 in patients of novel coronavirus disease. Clin Infect Dis [published online ahead of print]

Long Q X, Tang X J, Shi Q L, et al. (2020) Clinical and immunological assessment of asymptomatic SARS-CoV2 infections Nat Med 26 1200-1204

Mercatelli D and Giorgi F M (2020) Geographic and Genomic Distribution of SARS-CoV-2 Mutations Front Microbiol 111800

Brufsky A (2020) Distinct viral clades of SARS-CoV-2: Implications for modeling of viral spread $J$ Med Virol [published online ahead of print]

Coronaviridae Study Group of the International Committee on Taxonomy of Viruses. The species Severe acute respiratory syndrome-related coronavirus: classifying 2019-nCoV and naming it SARS-CoV-2 (2020) Nat Microbiol 5 536-544

Forster P, Forster L, Renfrew C and Forster M (2020) Phylogenetic network analysis of SARS-CoV-2 genomes it does no one knows how long that might take. Shall we wait for these people to get infected and attain immunity to be able to get certified? This might also lead to discrimination.

Thus, instead of depending solely on naturally obtained immunity which might occur in due course, it is better to invest time, effort and money to develop carefully designed vaccine candidates showing cross neutralizing properties which will be applicable universally to every immuno-competent people getting vaccinated. Also, antiviral candidates or drug repurposing could be another area where scientists are already focussing and warrants greater focus.

\section{Acknowledgements}

We thank CSIR and AcSIR for academic support.

Proc Natl Acad Sci U S A 117 9241-9243

Halstead S B (2003) Neutralization and antibody-dependent enhancement of dengue viruses Adv Virus Res 60 421-467

Halstead S B and O'Rourke E J (1977) Antibody-enhanced dengue virus infection in primate leukocytes Nature 265 739-741

Rothman A L (2011) Immunity to dengue virus: a tale of original antigenic sin and tropical cytokine storms Nat Rev Immunol $11532-543$

Arvin A M, Fink K, Schmid M A, et al. (2020) A perspective on potential antibody-dependent enhancement of SARS-CoV2 Nature 584 353-363

GBD (2015) Disease and Injury Incidence and Prevalence Collaborators. Global, regional, and national incidence, prevalence, and years lived with disability for 310 diseases and injuries, 1990-2015: a systematic analysis for the Global Burden of Disease Study 2015 [published correction appears in Lancet. 2017 Jan 7; 389(10064):e1]. Lancet. 2016; 388 1545-1602

Grifoni A, Weiskopf D, Ramirez S I, et al. (2020) Targets of T Cell Responses to SARS-CoV-2 Coronavirus in Humans with COVID-19 Disease and Unexposed Individuals Cell 181 1489-1501

Immunity Passports' for Covid-19 May Lead to Discrimination, Intentional Infections: Scientists. The Times of India. 